\title{
Correction to: Epidemiological, otolaryngological, olfactory and gustatory outcomes according to the severity of COVID-19: a study of 2579 patients
}

\author{
Jerome R. Lechien ${ }^{1,2,3,4}$ (1) - Carlos M. Chiesa-Estomba ${ }^{1,5} \cdot$ Luigi A. Vaira $^{6}$ - Giacomo De Riu ${ }^{6}$. Giovanni Cammaroto ${ }^{1,7}$. \\ Younes Chekkoury-Idrissi ${ }^{3} \cdot$ Marta Circiu $^{3} \cdot$ Lea Distinguin ${ }^{3} \cdot$ Fabrice Journe $^{2} \cdot$ Christophe de Terwangne $^{8}$. \\ Shahram Machayekhi ${ }^{8} \cdot$ Maria R. Barillari ${ }^{1,9} \cdot$ Christian Calvo-Henriquez $^{10} \cdot$ Stéphane Hans $^{3} \cdot$ Sven Saussez ${ }^{1,2,4}$
}

Published online: 10 February 2021

c) Springer-Verlag GmbH Germany, part of Springer Nature 2021

\section{Correction to: European Archives of Oto-Rhino-Laryngology https://doi.org/10.1007/s00405-020-06548-w}

In the original publication of the article, one of the coauthor's name was published incorrectly as "Liugi A. Varia". The correct name should read as "Luigi A. Vaira".

The original article has been updated.

Publisher's Note Springer Nature remains neutral with regard to jurisdictional claims in published maps and institutional affiliations.

The original article can be found online at https://doi.org/10.1007/ s00405-020-06548-w.

Jerome R. Lechien

Jerome.Lechien@umons.ac.be

1 COVID-19 Task Force of the Young-Otolaryngologists of the International Federations of Oto-rhino-laryngological Societies (YO-IFOS), Paris, France

2 Department of Human Anatomy and Experimental Oncology, Faculty of Medicine, UMONS Research Institute for Health Sciences and Technology, University of Mons (UMons), Mons, Belgium

3 Department of Otolaryngology-Head and Neck Surgery, Foch Hospital, School of Medicine, UFR Simone Veil, Université Versailles Saint-Quentin-en-Yvelines (Paris Saclay University), Paris, France

4 Department of Otorhinolaryngology and Head and Neck Surgery, CHU de Bruxelles, CHU Saint-Pierre, School of Medicine, Université Libre de Bruxelles, Brussels, Belgium
5 Department of Otorhinolaryngology-Head and Neck Surgery, Hospital Universitario Donostia/ Biodonostia Research Institute, San Sebastian, Spain

6 Maxillofacial Surgery Unit, University Hospital of Sassari, Sassari, Italy

7 Department of Otolaryngology-Head and Neck Surgery, Morgagni Pierantoni Hospital, Forli, Italy

8 Intensive Care Unit, EpiCURA Hospital, Hornu, Belgium

9 Division of Phoniatrics and Audiology, Department of Mental and Physical Health and Preventive Medicine, University of Naples SUN, Naples, Italy

10 Department of Otorhinolaryngology and Head and Neck Surgery, Hospital Complex of Santiago de Compostela, Santiago de Compostela, Spain 